\title{
Low prevalence of gingival overgrowth associated to new imunossupressive protocols with cyclosporin
}

\section{Luiz Augusto Wentz(a) Sara Cioccari Oliveira ${ }^{(a)}$ Carlos Heitor Cunha Moreira ${ }^{(b)}$ Cassiano Kuchenbecker Rösing ${ }^{(c)}$}

(a) Periodontics, School of Dentistry, Federal University of Santa Maria, Santa Maria, RS, Brazil.

(b) Department of Stomatology, School of Dentistry, Federal University of Santa Maria, Santa Maria, RS, Brazil.

(c) Departament of Periodontics, School of Dentistry, Universidade Federal do Rio Grande do Sul, Porto Alegre, RS, Brazil.

Declaration of Interests: The authors certify that they have no commercial or associative interest that represents a conflict of interest in connection with the manuscript.

Corresponding author:

Cassiano Kuchenbecker Rösing

E-mail: ckrosing@hotmail.com

Received for publication on Jun 01, 2011 Accepted for publication on Aug 30, 2011

\begin{abstract}
Gingival overgrowth $(\mathrm{GO})$ is a frequent finding in patients treated with cyclosporine (CsA). This study investigated the prevalence and severity of GO in patients who received kidney transplant and CsA therapy, as well as associations with pharmacological and clinical factors. This cross-sectional study included 63 kidney transplant recipients who were treated with CsA in a university hospital. Demographic, pharmacological, and periodontal data were collected. The primary variable was GO. Independent sample $t$ - and chi-square tests were used to compare means in groups with versus without GO. The response rate was $86.3 \%$. Overall, $40 \%$ of patients had some degree of GO. Eleven individuals presented GO scores $>10 \%$, and 5 individuals reached $30 \%$. The mean GO percentage was low $(6.79 \pm 15.83)$. Patients that were concurrently under nifedipine treatment showed a non-significant trend toward a greater prevalence of GO. Mean CsA dosage and serum levels were $3.20 \pm 0.94 \mathrm{mg} / \mathrm{kg} / \mathrm{d}$ and $156.12 \pm 162.75 \mathrm{ng} / \mathrm{mL}$, respectively. There were no statistically significant differences between patients with versus without GO nor between the groups receiving nifedipine, no drug, or verapamil. The GO prevalence and severity rates were lower than those reported in previous studies and seemed to be independent of drug interactions.
\end{abstract}

Descriptors: Oral Medicine; Gingival Overgrowth; Gingival Diseases; Epidemiologic Studies; Kidney Transplantation.

\section{Introduction}

Gingival overgrowth $(\mathrm{GO})$ is a frequent adverse effect in patients who receive kidney transplant and undergo immunosuppression with cyclosporine-A (CsA). ${ }^{1,2}$ Since the introduction of CsA in the 1980s, transplant success and organ survival rates have increased significantly. ${ }^{3}$ Solid organ and tissue transplants have been developed, and CsA remains a widely used drug. However, CsA may have damaging side effects, such as nephrotoxicity, hepatotoxicity, hypertension, and gingival overgrowth (GO). ${ }^{1,4}$ GO is a distressing and disfiguring condition that can affect speech, mastication, oral hygiene, and aesthetics. ${ }^{4}$ The prevalence of GO ranges from $25 \%$ to $81 \%$, depending on the study population, index, doses, serum levels, treatment duration, and interactions with concurrently administered drugs. ${ }^{5,6}$

Mechanisms proposed to explain the occurrence and distribution 
of GO point to a multifactorial model. Other factors seem to be associated with GO and cyclosporine therapy. Several authors have studied the etiology of GO in patients receiving kidney transplant surgery and immunosuppressive treatment. Studies have evaluated the association of GO with bacterial plaque, ${ }^{7}$ periodontal disease, treatment dosage and duration, plasma concentrations, ${ }^{5}$ concurrent use of calcium channel blockers (CCBs), ${ }^{8}$ and genetic susceptibility. ${ }^{9}$ Greenberg et al. ${ }^{3}$ recently studied a sample of 115 patients that underwent kidney transplants and found a GO prevalence of 53\% among those who were treated with CsA. De Oliveira Costa et al. ${ }^{8}$ did not find any association of GO with demographic or pharmacological factors. Only papillary bleeding index, azathioprine dose, and concurrent treatment with CCBs were significantly associated with GO prevalence and severity.

Current immunosuppressive protocols define rules for prescribing specific immunosuppressants and their dosages. The goal of these new protocols is to decrease the CsA plasma concentration as much as possible without losing the desired immunosuppression. At the core of these protocols is the concept of maximizing effect while minimizing risk. The aim of the present study was to determine whether the periodontal condition of patients who have undergone kidney transplants is associated with the drugs used in their treatments.

\section{Methodology Study population}

This cross-sectional study was conducted in an outpatient nephrology service of a university hospital from January to September 2009. All eligible kidney transplant recipients who were seen in this service were selected to participate in the study ( $\mathrm{n}=73$ ). Patients were seen regularly to control pharmacological treatments and to monitor organ survival. Eligibility criteria were:

- transplant at least 6 months before the study,

- age of $\geq 18$ years at the time of the study,

- immunosuppressive treatment with CsA for $\geq 6$ months,

- presence of $\geq 6$ of the 12 anterior teeth, and

- no periodontal treatment in the 6 months prior to examination.

During the 9 months of data collection, patients that met inclusion criteria were invited to participate in the study. Evaluations were made on the day when the routine medical visit was scheduled and according to the availability of selected participants.

This study was approved by the Ethics in Research Committee of the Federal University of Santa Maria, Brazil. All patients signed an informed consent form.

\section{Data collection}

Eligible patients answered a structured questionnaire and underwent a clinical examination performed by 2 trained and calibrated examiners. Kappa values $( \pm 1 \mathrm{~mm})$ for probing pocket depth (PPD) and clinical attachment loss were 0.98 and 0.89 , respectively, for examiner 1 (LAW); and 0.95 and 0.91 , respectively, for examiner 2 (SCO). The inter-examiner agreements were 0.94 and 0.78 , respectively.

All teeth, except third molars, were examined. The following clinical variables were collected: plaque index (PI), ${ }^{10}$ gingival index (GI),${ }^{11}$ and plaque retention factors (PRF) at 4 sites per tooth. Bleeding on probing (BOP), PPD, and clinical attachment level (CAL) were evaluated at 6 sites with a manual periodontal probe (PCP UNC 15 Trinity, São Paulo, Brazil). Patients were interviewed to obtain demographic, behavioral, and medical information. Medical charts were reviewed to check the veracity of information provided by patients. The evaluation and classification of GO severity were made by the same examiners via visual inspection and were recorded. Scores for GO were based on the index described by Seymour et al., ${ }^{12}$ for which the examiners were trained.

Medical and pharmacological data were retrieved from the clinical records (i.e., medical charts) for each participant. Charts were carefully examined and data were confirmed with the medical team responsible for the patient. Data entered on the most recent date were used for the analysis.

With respect to the use of CCB, the patients were divided into two groups: one that used nifedipine (a 
drug associated with GO) and another that received verapamil or other non-CCB drugs.

\section{Statistical analysis}

Data were analyzed through the SPSS 13.0 software package (SPSS Inc., Chicago, USA). Descriptive periodontal data (PI, GI, PRF, PPD, CAL, and BOP) were calculated and evaluated according to their mean values and standard deviations. Demographic, behavioral, and transplantation data were described according to absolute values and corresponding percentages. The GO prevalence was calculated for patients that received transplants and was classified into groups according to mean percentage. The GO groups were compared according to their CsA plasma levels and doses with an independent $t$-test. The association between GO groups and CCB use was evaluated with the chi-square test. The level of significance was set at $5 \%$.

\section{Results}

From among the 102 kidney transplant recipients who were regularly seen at the Nephrology Outpatient Service of Santa Maria University Hospital, Brazil, 29 individuals were excluded from this study for the following reasons:

- no teeth $(\mathrm{n}=5)$,

$-<6$ teeth $(\mathrm{n}=3)$,

- not using CsA ( $\mathrm{n}=16)$, and

- transplant performed $<6$ months before start of the study $(\mathrm{n}=5)$.

Of the remaining 73 patients that met inclusion criteria, 10 did not participate in the study. Six patients answered the questionnaire, but did not undergo the oral examination for a variety of reasons:

- transportation difficulties,

- health problems that prevented examination, and

- refusal to participate in examination.

Four patients could not be contacted, although several attempts were made to reach them. Therefore, 63 patients were contacted, underwent the full oral examination, and answered the questionnaire. The study population is described in Table 1.

Most participants in the study were men $(\mathrm{n}=39)$
Table 1 - Demographic, behavioral, and transplantation data $(n=63)$.

\begin{tabular}{|c|c|c|}
\hline Variable & $n^{*}$ & $\%$ \\
\hline Age (years; mean $\pm S D$ ) & $44.8 \pm 13.3$ & \\
\hline \multicolumn{3}{|l|}{ Gender } \\
\hline - Male & 39 & 61.9 \\
\hline - Female & 24 & 38.1 \\
\hline \multicolumn{3}{|l|}{ Skin color } \\
\hline - White & 48 & 76.2 \\
\hline - Nonwhite & 14 & 22.2 \\
\hline - Not reported & 1 & 1.6 \\
\hline \multicolumn{3}{|l|}{ Socioeconomic status } \\
\hline - High & 8 & 12.7 \\
\hline - Medium & 48 & 76.2 \\
\hline - Low & 7 & 11.1 \\
\hline Years of education (mean \pm SD) & $9.45 \pm 4.65$ & \\
\hline \multicolumn{3}{|l|}{ Smoking } \\
\hline - Nonsmoker & 37 & 58.7 \\
\hline - Smoker & 3 & 4.8 \\
\hline - Previous smoker & 23 & 36.5 \\
\hline \multicolumn{3}{|l|}{ First transplantation } \\
\hline - Yes & 60 & 95.2 \\
\hline - No & 3 & 4.8 \\
\hline Time since transplant (years; mean \pm SD) & $6.91 \pm 5.13$ & \\
\hline Number of teeth (mean \pm SD) & $21.53 \pm 6.41$ & \\
\hline \multicolumn{3}{|l|}{ Gingival bleeding self-report } \\
\hline - Yes & 28 & 44.4 \\
\hline - No & 35 & 55.6 \\
\hline \multicolumn{3}{|l|}{ Gingival overgrowth self-report } \\
\hline - Yes & 16 & 25.4 \\
\hline - No & 47 & 74.6 \\
\hline
\end{tabular}

unless otherwise indicated.

and white $(\mathrm{n}=48)$. Age ranged from 23 to 74 years. Most patients were middle class $(\mathrm{n}=48)$ and had finished elementary school. About $60 \%$ of the patients had never smoked $(\mathrm{n}=37)$. Mean number of teeth was 21.57 (range: 6 to 28). Gingival bleeding was reported by 28 patients, and about $75 \%$ of them did not perceive GO.

Data about the transplanted organ revealed that, up to the time the study was conducted, 60 patients had only 1 transplant, whereas 3 had $>1$ transplant 
due to rejection. Time from transplantation ranged from 6 months to 18 years. All but 1 patient underwent regular follow-ups at the nephrology outpatient service where the study was conducted, and the most prevalent consultation frequency was 3 months (range: 1 to 6 months). Table 2 describes the clinical periodontal conditions, frequency of GO, and drugs used.

Overall, $40 \%$ of patients had some degree of GO. The Seymour index ranged from 0 to 47 . The mean score for 11 patients was $>10 \%$. Only 5 patients $(8 \%)$ had a mean GO $>30 \%$. Mean PI, IG, and CAL values were not statistically different between patients with and without GO (cut-off point: $\geq 10 \%$ ).

All kidney transplant recipients in this study used CsA combined with other immunosuppressive drugs. Medication regimens included low-dose prednisone $(5 \mathrm{mg} / \mathrm{d})$ and a combination of CsA with mycophenolate mofetil (MMF) or azathioprine. Patients were divided into 2 groups according to CCB use:

1. CCB (nifedipine) and

2. Not taking CCB (or verapamil).

About $75 \%$ of patients were in the second group, and 17 patients used nifedipine concurrently. The mean CsA dose was $3.20 \pm 0.94 \mathrm{mg} / \mathrm{kg} / \mathrm{d}$ (range: 1.57 to $6 \mathrm{mg} / \mathrm{kg} / \mathrm{d})$. The mean ( $\pm \mathrm{SD}$ ) and median CsA serum levels were $156.12 \pm 162.75 \mathrm{ng} / \mathrm{mL}$ and $121 \mathrm{ng} / \mathrm{mL}$, respectively. There were no statistically significant differences in CsA dose $(\mathrm{mg} / \mathrm{kg} / \mathrm{d})$ or serum levels $(\mathrm{ng} / \mathrm{mL})$ between patients not taking any CCB or verapamil and patients taking nifedipine (Table 3).

When the CsA serum levels and daily doses (mg/ $\mathrm{kg} / \mathrm{d}$ ) were compared in the analysis of the presence of absence of GO, no statistically significant dif- ferences were found between the groups. A greater prevalence of GO was found among patients that used nifedipine, but the difference was not statistically significant (Table 3).

No statistically significant differences in PI or GI were observed between the groups of patients with $\mathrm{GO}<10 \%$ versus those with $\mathrm{GO} \geq 10 \%$ (Figure 1 ). Inflammatory indices (PPD and BOP) were elevated in the group of patients with GO. For individuals with $\mathrm{GO}<10 \%$ or $\geq 10 \%$, the mean PPD values were $2.04 \mathrm{~mm}$ and $3.27 \mathrm{~mm}$ and the mean BOP percentages were $9.39 \%$ and $42.41 \%$, respectively. For both parameters, statistically significant differences $(p<0.05)$ were observed.

Table 2 - Evaluation of gingival overgrowth, periodontal conditions, and drug interactions in kidney transplant individuals $(n=63)$.

\begin{tabular}{|c|c|c|}
\hline Variable & Mean \pm SD & $\%$ \\
\hline$\%$ GO score & $6.79 \pm 15.83$ & \\
\hline \multicolumn{3}{|l|}{ Mean GO strata } \\
\hline - Zero & & 60.3 \\
\hline - 1-9.99 & & 22.2 \\
\hline$\cdot \geq 10$ & & 17.5 \\
\hline $\mathrm{Pl}$ & $0.88 \pm 0.56$ & \\
\hline $\mathrm{Gl}$ & $0.84 \pm 0.53$ & \\
\hline$\%$ PRF & $46.5 \pm 32.32$ & \\
\hline PPD & $2.26 \pm 0.82$ & \\
\hline CAL & $2.06 \pm 1.39$ & \\
\hline$\% \mathrm{BOP}$ & $15.15 \pm 19.14$ & \\
\hline \multicolumn{3}{|l|}{$\mathrm{CCB}$} \\
\hline - Nifedipine & & 27 \\
\hline - Verapamil or (not receiving CCB) & & 73 \\
\hline
\end{tabular}

PI (plaque index); GI (gingival index); PRF (plaque retention factors); PPD (periodontal pocket depth); CAL (clinical attachment level); BOP (bleeding on probing); CCB (calcium channel blocker).
Table 3 - Association between dose and CsA plasma levels, drug interactions, and $\mathrm{GO}$ according to severity.

\begin{tabular}{c|c|c|c}
\hline Parameter & $\begin{array}{c}<10 \% \text { mean } \\
\text { percentage } \\
\text { Plasma level of CsA }(\mathrm{ng} / \mathrm{mL}) \times \mathrm{GO}\end{array}$ & $\begin{array}{c}\geq 10 \% \text { mean } \\
\text { percentage }\end{array}$ & $P$ \\
\hline CsA dosage $(\mathrm{mg} / \mathrm{kg} / \mathrm{d}) \times \mathrm{GO}$ & $3.22 \pm 0.98 \pm 177.88$ & $119.54 \pm 29.8$ & $0.41^{*}$ \\
\hline CCB $($ nifedipine) & 76.5 & $3.12 \pm 0.73$ & $0.75^{*}$ \\
\hline Not taking CCB (or verapamil) & 84.8 & 23.5 & \\
\hline * Independent sample t-test; ${ }^{* *}$ Chi-square. &
\end{tabular}




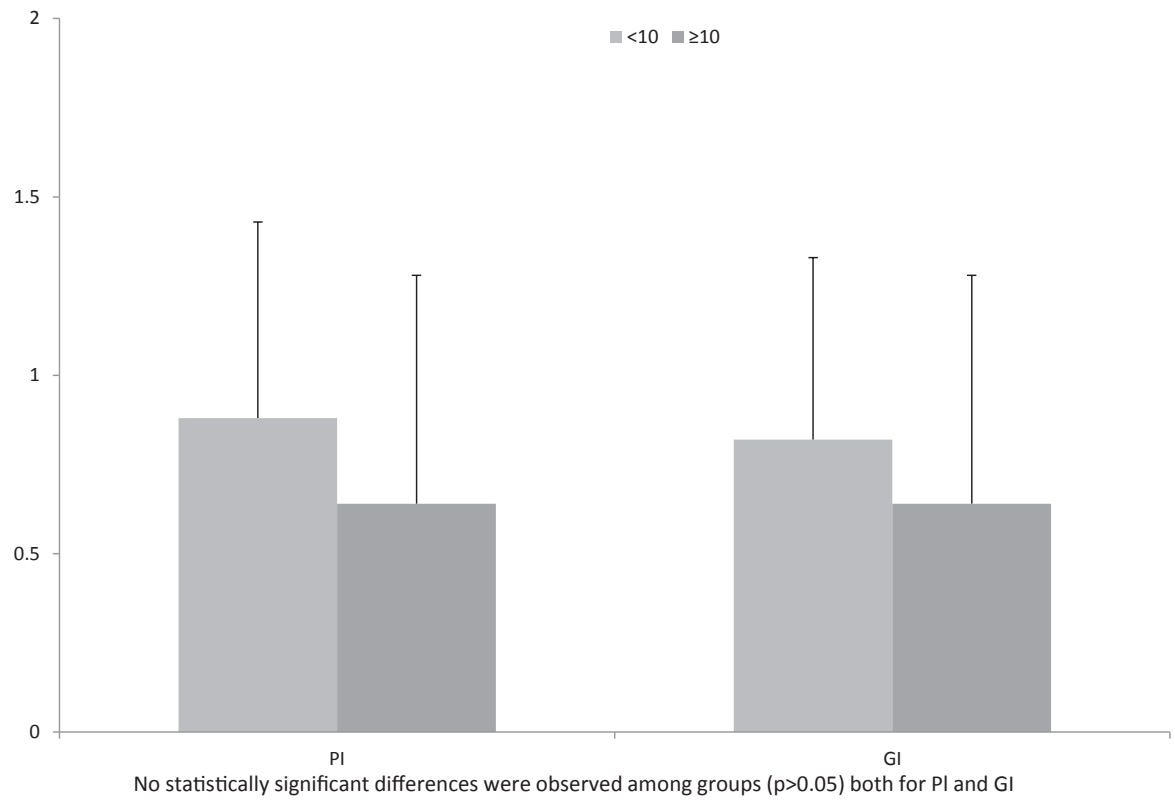

Figure 1 - Mean scores and standard deviation values of $\mathrm{Pl}$ and Gl for patients with $\mathrm{GO}<10 \%$ and $\mathrm{GO} \geq 10 \%$.

\section{Discussion}

A $40 \%$ GO prevalence and mean severity of $6.79 \%$ were found in kidney transplant recipients treated with CsA, with or without CCB. This result may be explained by the use of novel immunosuppression protocols, whose effects seem to be dose-dependent and reversible with lower doses, ${ }^{13,14}$ although several studies have failed to confirm this association. ${ }^{15,16}$ By adjusting the CsA dosage for each patient, ${ }^{17}$ current protocols ensure that CsA dosages are lower than those administered when the drug was first introduced in the market.

In the present study, the use of antibiotics or anti-inflammatory drugs was not considered an exclusion criterion because these drugs normally have a time-limited effect and do not directly influence the main outcome, GO. Previous immunosuppressive regimens used oral dosages of 10 to $20 \mathrm{mg} / \mathrm{kg} / \mathrm{d}$ during the organ maintenance phase. ${ }^{5}$ The mean CsA dosage in this study was $3.2 \mathrm{mg} / \mathrm{kg} / \mathrm{d}$ (or $220 \mathrm{mg} / \mathrm{d}$ ). This value is consistent with that reported in some previous studies, ${ }^{8,16}$ but less than the median values reported in Thomason et al.'s ${ }^{15}$ study $(350 \mathrm{mg} / \mathrm{d}$ for CsA + CCB group, and $300 \mathrm{mg} /$ day for CsA group).

Somacarrera et al. ${ }^{18}$ concluded that CsA serum level is the most important factor in GO severity. Among the transplant recipients tested, kidney transplant recipients had the lowest CsA levels and significantly less GO. This observation might be associated with the fact that these patients had lower CsA blood concentrations (260-340 ng/mL) than heart transplant recipients $(550-670 \mathrm{ng} / \mathrm{mL})$. In our study, the mean CsA blood concentration was $156 \mathrm{ng} / \mathrm{ml}$, which is similar to values reported in recent studies. ${ }^{8,16}$ We found no association between the CsA serum levels and dosages in patients with or without GO. These results may be explained by the fact that the CsA doses were low. ${ }^{2}$

Various values for the prevalence of GO in patients treated with CsA have been reported. Thomason et al. ${ }^{15}$ found a GO prevalence of $47.82 \%$ (scores $\geq 30 \%$ ) when CsA and nifedipine were used in combination, and $37.5 \%$ for CsA alone. The results reported by de Oliveira Costa et al. ${ }^{8}$ and Paixão et al. ${ }^{19}$ in Brazil revealed significant GO (score $\geq 30 \%$ ) in $38.1 \%$ and $17.4 \%$ of CsA-treated patients, respectively. Our study found a lower percentage $(8 \%)$ of patients using the same GO cut-off; when the cut-off point was lowered to $>10 \%$, the GO prevalence was $17.5 \%$.

Daley et al. ${ }^{20}$ reported that $70 \%$ of patients evaluated at 2.5 years had GO. Their results suggest that $\mathrm{GO}$ progresses for months and reaches a plateau after 1 year of treatment with CsA. Different attempts were made to establish the possible influence of time in the prevalence of GO. In our study, GO preva- 
lence did not differ with time since transplantation or CsA treatment duration (data not shown). A possible explanation for these negative findings may lie in the inclusion criteria of the study, which stipulated a minimum CsA use time of 6 months (which is sufficient time for GO to occur).

The relationship between dental plaque and GO is controversial. Tyldesley and Rotter, ${ }^{4}$ Thomason et al., ${ }^{15}$ and Greenberg et al. ${ }^{3}$ have suggested that GO results from inadequate plaque control. Abundant plaque ( $\mathrm{PI} \geq 2$ in $40 \%$ of the sites) was associated with a 5.4 times higher risk of GO. ${ }^{3}$ However, according to Seymour and Smith, ${ }^{7}$ optimal plaque control is insufficient to prevent GO. Our results showed low mean PI (0.88) and GI (0.84) values, and did not reveal any association between these parameters and the occurrence of GO.

With regard to calcium antagonist drugs, nifedipine has been associated with GO more often than verapamil (with prevalence rates of $6-15 \%$ and $<5 \%$, respectively). ${ }^{14}$ Verapamil has been reported to have little effect on the prevalence or severity of cyclosporine-induced GO. ${ }^{21}$ In this study, patients that used nifedipine showed a non-significant trend toward greater GO scores.

One possible limitation of the present study is that the number of patients in each group was not as high as desired. However, we attempted to examine all individuals from the hospital service, with a census characteristic. The non-significant trends observed here might become significant effects in a larger multi-institutional study. However, inclusion of individuals from other transplantation centers could increase other sources of bias.

The GO prevalence results reported here are in agreement with those reported in other studies. ${ }^{8,15}$ Greenberg et al. ${ }^{3}$ found that GO prevalence was greater among patients that used CsA and CCB $(76 \%)$ than among patients that did not use those

\section{References}

\footnotetext{
1. Rateitschak-Pluss EM, Hefti A, Lortscher R, Thiel G. Initial observation that cyclosporin-A induces gingival enlargement in man. J Clin Periodontol. 1983 May;10(3):237-46.

2. Thomason JM, Seymour RA, Ellis JS. Risk factors for gingi-
}

drugs $(13 \%)$. The GO severity was also increased among the group that used CsA and CCB. The increase in GO scores may be a consequence of the synergistic effect with CCB drugs. ${ }^{15}$ In our study, inflammatory parameters, such as PPD and BOP, were greater in the group of patients with GO (> 10\%). In a recent study, Guo et al. ${ }^{22}$ used ligatures to induce inflammation in rats receiving treatment with or without CsA. They concluded that CsA-induced GO may be exacerbated by local inflammation. However, a double association may be present: GO, which generates a greater PPD, also produces a more favorable environment for inflammation.

There was an agreement between self-reported $\mathrm{GO}$ and mean GO > 10\% among our patients. Although GO severity was not high, it seemed to be serious enough to be perceived by patients.

Most studies that have examined the effects of CsA on GO have been cross-sectional, and one of the limitations of such studies has been that causality cannot be inferred. However, the present study design did define the presence and severity of GO in patients after the initiation of immunosuppressive treatments, which may suggest associations with clinical and pharmacological parameters. In our study, methods were carefully controlled to reduce bias. The response rate was high $(86.30 \%)$, and examiners were trained and calibrated. In addition, access to medical charts provided data to confirm the doses used by the patients.

\section{Conclusion}

New pharmacological protocols for the use of CsA seem to result in lower GO prevalence and severity, regardless of interactions with other drugs. Despite these results, measures should be taken to protect the periodontal health of patients using CsA after transplantation.

val overgrowth in patients medicated with ciclosporin in the absence of calcium channel blockers. J Clin Periodontol. 2005 Mar;32(3):273-9.

3. Greenberg KV, Armitage GC, Shiboski CH. Gingival en- 
largement among renal transplant recipients in the era of new-generation immunosuppressants. J Periodontol. 2008 Mar;79(3):453-60.

4. Tyldesley WR, Rotter E. Gingival hyperplasia induced by cyclosporin-A. Br Dent J. 1984 Nov 10;157(9):305-9.

5. Seymour RA, Jacobs DJ. Cyclosporin and the gingival tissues. J Clin Periodontol. 1992 Jan;19(1):1-11.

6. Spratt H, Boomer S, Irwin CR, Marley JJ, James JA, Maxwell $\mathrm{P}$, et al. Cyclosporin associated gingival overgrowth in renal transplant recipients. Oral Dis. 1999 Jan;5(1):27-31.

7. Seymour RA, Smith DG. The effect of a plaque control programme on the incidence and severity of cyclosporin-induced gingival changes. J Clin Periodontol. 1991 Feb;18(2):107-10.

8. de Oliveira Costa F, Diniz Ferreira S, de Miranda Cota LO, da Costa JE, Aguiar MA. Prevalence, severity, and risk variables associated with gingival overgrowth in renal transplant subjects treated under tacrolimus or cyclosporin regimens. J Periodontol. 2006 Jun;77(6):969-75.

9. Drozdzik M, Kurzawski M, Drozdzik A, Kotrych K, Banach J, Pawlik A. Interleukin-6 gene polymorphism in renal transplant patients with and without gingival overgrowth. J Clin Periodontol. 2005 Sep;32(9):955-8.

10. Silness J, Loe H. Periodontal disease in pregnancy. II. Correlation between oral hygiene and periodontal condtion. Acta Odontol Scand. 1964 Feb;22:121-35.

11. Loe H. The Gingival Index, the Plaque Index and the Retention Index Systems. J Periodontol. 1967 NovDec;38(6):Suppl:610-6.

12. Seymour RA, Smith DG, Turnbull DN. The effects of phenytoin and sodium valproate on the periodontal health of adult epileptic patients. J Clin Periodontol. 1985 Jul;12(6):413-9.

13. Hallmon WW, Rossmann JA. The role of drugs in the pathogenesis of gingival overgrowth. A collective review of current concepts. Periodontol 2000. 1999 Oct;21:176-96.
14. Dongari-Bagtzoglou A, Research, Science and Therapy Committee, American Academy of Periodontology. Drug-associated gingival enlargement. J Periodontol. 2004 Oct;75(10):1424-31.

15. Thomason JM, Seymour RA, Rice N. The prevalence and severity of cyclosporin and nifedipine-induced gingival overgrowth. J Clin Periodontol. 1993 Jan;20(1):37-40.

16. Vescovi P, Meleti M, Manfredi M, Merigo E, Pedrazzi G. Cyclosporin-induced gingival overgrowth: a clinical-epidemiological evaluation of 121 Italian renal transplant recipients. J Periodontol. 2005 Aug;76(8):1259-64.

17. Seymour RA, Ellis JS, Thomason JM. Risk factors for druginduced gingival overgrowth. J Clin Periodontol. 2000 Apr;27(4):217-23.

18. Somacarrera ML, Hernandez G, Acero J, Moskow BS. Factors related to the incidence and severity of cyclosporin-induced gingival overgrowth in transplant patients. A longitudinal study. J Periodontol. 1994 Jul;65(7):671-5.

19. Paixao CG, Sekiguchi RT, Saraiva L, Pannuti CM, Silva HT, Medina-Pestana JO, et al. Gingival overgrowth among patients medicated with cyclosporin A and tacrolimus undergoing renal transplantation: a prospective study. J Periodontol. $2011 \mathrm{Feb} ; 82(2): 251-8$.

20. Daley TD, Wysocki GP, Day C. Clinical and pharmacologic correlations in cyclosporine-induced gingival hyperplasia. Oral Surg Oral Med Oral Pathol. 1986 Oct;62(4):417-21.

21. Cebeci I, Kantarci A, Firatli E, Carin M, Tuncer O. The effect of verapamil on the prevalence and severity of cyclosporineinduced gingival overgrowth in renal allograft recipients. $\mathrm{J}$ Periodontol. 1996 Nov;67(11):1201-5.

22. Guo J, Wang W, Yao L, Yan F. Local inflammation exacerbates cyclosporine a-induced gingival overgrowth in rats. Inflammation. 2008 Dec;31(6):399-407. 